\title{
REGULARITY OF SOAP FILM-LIKE SURFACES SPANNING GRAPHS IN A RIEMANNIAN MANIFOLD
}

\author{
Robert Gulliver, Sung-Ho Park, Juncheol Pyo, and Keomkyo Seo
}

\begin{abstract}
Let $M$ be an $n$-dimensional complete simply connected Riemannian manifold with sectional curvature bounded above by a nonpositive constant $-\kappa^{2}$. Using the cone total curvature $T C(\Gamma)$ of a graph $\Gamma$ which was introduced by Gulliver and Yamada [8], we prove that the density at any point of a soap film-like surface $\Sigma$ spanning a graph $\Gamma \subset M$ is less than or equal to $\frac{1}{2 \pi}\left\{T C(\Gamma)-\kappa^{2} \operatorname{Area}(p \circledast \Gamma)\right\}$. From this density estimate we obtain the regularity theorems for soap film-like surfaces spanning graphs with small total curvature. In particular, when $n=3$, this density estimate implies that if

$$
T C(\Gamma)<3.649 \pi+\kappa^{2} \inf _{p \in M} \operatorname{Area}(p \circledast \Gamma),
$$

then the only possible singularities of a piecewise smooth $(\mathbf{M}, 0, \delta)$-minimizing set $\Sigma$ are the $Y$-singularity cone. In a manifold with sectional curvature bounded above by $b^{2}$ and diameter bounded by $\pi / b$, we obtain similar results for any soap film-like surfaces spanning a graph with the corresponding bound on cone total curvature.
\end{abstract}

\section{Introduction}

In 2002, Ekholm, White, and Wienholtz [6] ingeniously proved that in an $n$-dimensional Euclidean space, classical minimal surfaces of arbitrary topological type bounded by a Jordan curve with total curvature at most $4 \pi$ must be smoothly embedded. One year later, Choe and Gulliver [5] extended the Ekholm-White-Wienholtz result to minimal surfaces in an $n$-dimensional complete simply connected Riemannian manifold $M$ with sectional curvature bounded above by a nonpositive constant $-\kappa^{2}$. More precisely, they proved that if $\Gamma$ is a Jordan curve in $M$ with total curvature

$$
T C(\Gamma):=\int_{\Gamma}|\vec{k}| d s \leq 4 \pi+\kappa^{2} \inf _{p \in M} \operatorname{Area}(p \circledast \Gamma)
$$

Received December 19, 2008; Revised November 3, 2009.

2000 Mathematics Subject Classification. 58E35, 49Q20.

Key words and phrases. soap film-like surface, graph, density.

The second author was supported by Hankuk University of Foreign Studies Research Fund of 2010. The third author was supported by grant Proj. No. R17-2008-001-01001-0 from NRF. 
then every branched minimal surface bounded by $\Gamma$ is embedded. Here $p * \Gamma$ is the geodesic cone, which is the union of the geodesic segments $\overline{p q}$ from $p$ to $q$ over all $q \in \Gamma$. Moreover they proved a similar theorem for minimal surfaces in the hemisphere $([5])$.

For nonclassical minimal surfaces, Ekholm, White, and Wienholtz also showed that for a given soap film-like surface $\Sigma$ with a simple closed boundary curve $\Gamma$ and with density at least one at every point on the support of $\Sigma \backslash \Gamma$, the condition $T C(\Gamma) \leq 3 \pi$ implies that $\Sigma$ is smooth in the interior. Recall that the density of a surface $\Sigma$ at a point $p \in M$ is defined to be

$$
\Theta(\Sigma, p)=\lim _{\varepsilon \rightarrow 0} \frac{\operatorname{Area}\left(\Sigma \cap B_{\varepsilon}(p)\right)}{\pi \varepsilon^{2}},
$$

where $B_{\varepsilon}(p)$ is the geodesic ball of $M$ with radius $\varepsilon$, centered at $p$. Recently, Gulliver and Yamada [8] proved that similar regularity theorems hold for soap film-like surfaces spanning graphs in $\mathbb{R}^{n}$

A graph $\Gamma$ is a finite union of closed arcs $a_{i}$ meeting at vertices $q_{j}$, each of which has valence at least 2 . Here the valence of a vertex $q$ is defined by the number of times $q$ occurs as an end point among all of the 1-simplices $a_{i}$. We assume that each $a_{i}$ is $C^{2}$ and meets its end points with $C^{1}$ smoothness. As in [8], we define the contribution to cone total curvature $t c(q)$ at a vertex $q$ by

$$
t c(q):=\sup _{e \in T_{q}(M),\|e\|=1}\left\{\sum_{a_{k}: q \in a_{k}}\left(\frac{\pi}{2}-L_{q}\left(T_{k}(q), e\right)\right)\right\},
$$

where $\angle_{q}\left(T_{k}(q), e\right)$ is the angle between the tangent vector $T_{k}(q)$ pointing into $a_{k}$ at $q$ and the direction $e$. If $\Gamma$ is a piecewise smooth Jordan curve, then the contribution to cone total curvature $t c(q)$ at $q$ is nothing but the exterior angle at the vertex $q$ : in fact, the supremum is assumed for $e$ in the shorter great-circle arc of $S^{n-1}$ between the two tangent vectors at $q$. Define the cone total curvature $T C(\Gamma)$ of a graph $\Gamma$ by

$$
T C(\Gamma):=\int_{\Gamma^{\mathrm{reg}}}|\vec{k}| d s+\sum_{q \in V(\Gamma)} t c(q),
$$

where $V(\Gamma):=\{$ vertices of $\Gamma\}$ and $\Gamma^{\mathrm{reg}}:=\Gamma \backslash V(\Gamma)$.

In soap film experiments, one can construct soap films spanning graphs so that three surfaces meet along an edge or six surfaces meet at a point. Indeed there are only two possible singularities on area-minimizing soap filmlike surfaces in $\mathbb{R}^{3}$ (see [2]). The tangent cone to a soap film-like surface $\Sigma$ at a singularity is an area-minimizing cone: either the $Y$-singularity cone, which consists of three half-planes meeting at $120^{\circ}$, or the $T$-singularity cone which is spanned by the regular tetrahedron with vertex at its center of mass. We will denote by $C_{Y}=3 / 2$ the density at its vertex of the $Y$-singularity cone and by $C_{T}=\frac{3}{\pi} \arccos \left(-\frac{1}{3}\right) \approx 1.8245$ the density at its vertex of the $T$-singularity cone. 
Introducing a notion of cone total curvature $T C(\Gamma)$ for a graph $\Gamma$, that is, a finite 1-dimensional curved polyhedron, Gulliver and Yamada proved that for a soap film-like surface $\Sigma$ spanning a graph $\Gamma$ in $\mathbb{R}^{n}$, if $T C(\Gamma) \leq 2 \pi C_{Y}=3 \pi$, then $\Sigma$ is an embedded smooth surface or a subset of the $Y$-singularity cone with planar faces. Moreover they proved that if $T C(\Gamma) \leq 2 \pi C_{T}$, then a soap film-like surface $\Sigma$ spanning $\Gamma$ in $\mathbb{R}^{3}$ has possibly $Y$-singularities but no other singularities unless it is a subset of the $T$-singularity cone with planar faces. In this paper we extend the Gulliver-Yamada results to soap film-like surfaces spanning graphs in an $n$-dimensional Riemannian manifold $M$ with an upper bound on sectional curvature.

Let $\mathcal{S}_{\Gamma}$ be the collection of all immersed images $\Sigma=\cup \Sigma_{i}$ of a finite union of $C^{2}$-smooth open two-dimensional manifolds $\Sigma_{i}$ with compact closure, of class $C^{1}$ up to the piecewise $C^{1}$ boundary $\partial \Sigma_{i}$, so that $\Gamma \subset \cup \partial \Sigma_{i}$. Throughout this paper, we consider singular surfaces spanning an embedded graph $\Gamma$ in the class $\mathcal{S}_{\Gamma}$. In particular, $\Sigma \in \mathcal{S}_{\Gamma}$ is a rectifiable varifold [1]. A surface $\Sigma$ in $\mathcal{S}_{\Gamma}$ is said to be strongly stationary with respect to $\Gamma$ if the first variation of the area of $\Sigma$ is at most equal to the integral over $\Gamma$ of the length of the orthogonal component of the variational vector field normal to $\Gamma$ (see Definition 2.1 below and also [6], $[8]$ ). Unfortunately one cannot distinguish the boundary of a singular surface $\Sigma \in \mathcal{S}_{\Gamma}$ from $\cup \partial \Sigma_{i}$ in a classical sense. Moreover the definition of boundary motivated by Stokes' Theorem [7] is no longer valid (other than modulo two) because $\Sigma$ is not orientable in general. Therefore we define the boundary of $\Sigma \in \mathcal{S}_{\Gamma}$ in variational terms (see Definition 2.2 below).

Let $M$ be an $n$-dimensional complete simply connected Riemannian manifold with sectional curvature bounded above by a nonpositive constant $-\kappa^{2}$. We shall prove that if $\Gamma$ is an embedded graph in $M$ with

$$
T C(\Gamma) \leq 3 \pi+\kappa^{2}\left(\inf _{p \in M} \operatorname{Area}(p \circledast \Gamma)\right),
$$

then a soap film-like surface $\Sigma$ spanning $\Gamma$ is an embedded surface or a subset of the piecewise totally geodesic $Y$-singularity cone (Theorem 3.8). More precisely, the infimum of Area $(p * \Gamma)$ may be taken only over $p$ in the geodesic convex hull $\operatorname{Conv}(\Gamma)$ of $\Gamma$. Furthermore, we shall prove that if $\Gamma$ is a graph in $M^{3}$ with

$$
T C(\Gamma) \leq 2 \pi C_{T}+\kappa^{2}\left(\inf _{p \in \operatorname{Conv}(\Gamma)} \operatorname{Area}(p * \Gamma)\right),
$$

then the only possible singularities of a soap film-like surface $\Sigma$ in $M^{3}$ are the $Y$-singularity cone or, in special cases, the $T$-singularity cone (Theorem 3.9). Similar results for a manifold with bounded diameter, and the corresponding positive upper bound on sectional curvature, will be demonstrated in Section 4.

The key steps of the proofs of our theorems are as follows. We first compare the density $\Theta(\Sigma, p)$ of $\Sigma$ in $\mathcal{S}_{\Gamma}$ with the density $\Theta(p \circledast \Gamma, p)$ of the cone $p \nVdash \Gamma$ for $p \in \Sigma \backslash \Gamma$. This is related to the monotonicity property of minimal surfaces. Next, we construct a certain 2-dimensional cone with constant curvature 
metric locally isometric to the simply connected space form $\bar{M}^{2}$ of constant sectional curvature, which corresponds to $p \circledast \Gamma$ in $M$. With comparison results for geodesic curvature of cones and the Gauss-Bonnet Theorem for the cone, we shall obtain the above theorems.

\section{Preliminaries}

Let $\Gamma$ be a graph in an $n$-dimensional Riemannian manifold $M$, and let $\mathcal{S}_{\Gamma}$ be the class of singular surfaces as described in the introduction.

Definition 2.1 ([6]). A rectifiable varifold $\Sigma$ in $M$ is called strongly stationary with respect to $\Gamma$ if for all smooth variations $\phi: \mathbb{R} \times M \rightarrow M$ with $\phi(0, x) \equiv x$ we have

$$
\left.\frac{d}{d t}\right|_{t=0}(\operatorname{Area}(\phi(t, \Sigma))+\operatorname{Area}(\phi([0, t] \times \Gamma))) \geq 0 .
$$

Note that the strong stationarity of $\Sigma$ implies that $\Sigma \in \mathcal{S}_{\Gamma}$ is stationary. In fact, the first variation formula for area [11] gives

$$
\left.\frac{d}{d t}\right|_{t=0} \operatorname{Area}(\phi(t, \Sigma))=-\int_{\Sigma}\left\langle\vec{H}, X^{\perp}\right\rangle d A+\int_{\cup \partial \Sigma_{i}} \sum_{j \in J(p)}\left\langle\nu_{j}(p), X^{\perp}(p)\right\rangle d s,
$$

where $\vec{H}$ is a mean curvature vector of $\Sigma ; X^{\perp}$ is the normal component of the variational vector field $X=\frac{\partial \phi}{\partial t}(0, x) ; J(p)$ indexes the collection of surfaces $\Sigma_{j}$ which meet at a point $p$ in $\cup \partial \Sigma_{i}$; and $\nu_{j}(p)$ is the outward unit conormal vector to $\partial \Sigma_{j}$ along $\cup \partial \Sigma_{i}$, where surfaces $\Sigma_{j}$ meet for $j \in J(p)$.

If we take the variational vector field $X=\frac{\partial \phi}{\partial t}(0, x)$ to be supported in $M \backslash \cup \partial \Sigma_{i}$, then the stationarity of $\Sigma$ implies that the mean curvature vector $\vec{H} \equiv \overrightarrow{0}$ on the interior of each $\Sigma_{i}$. Furthermore, if we choose $X$ supported away from $\Gamma$, then it follows that

$$
\nu_{\Sigma}(p):=\sum_{j \in J(p) \subset I} \nu_{\Sigma_{j}}(p)=\overrightarrow{0}
$$

for almost all $p$ on $\cup \partial \Sigma_{i} \backslash \Gamma$, since the choice of $X$ is arbitrary along $\cup \partial \Sigma_{i} \backslash \Gamma$. We call the equation (2.1) the balancing of $\nu_{\Sigma_{i}}$ along the singular curves of $\Sigma$ away from $\Gamma[8$, p. 319].

It should be mentioned that the strong stationarity with respect to $\Gamma$ for a surface in $\mathcal{S}_{\Gamma}$ is equivalent to stationarity in $M \backslash \Gamma$ plus the following boundary condition.

Definition $2.2([6],[8])$. $\Gamma$ is said to be the variational boundary of a surface $\Sigma$ if there exists an $\mathcal{H}^{1}$ measurable vector field $\nu_{\Sigma}$ along $\Gamma$ which is orthogonal to $\Gamma$, with $\left|\nu_{\Sigma}\right| \leq 1$ a.e., such that for all smooth vector fields $X$ defined on $M$,

$$
\int_{\Sigma} \operatorname{div} X^{T} d A=\int_{\Gamma}\left\langle X, \nu_{\Sigma}\right\rangle d s
$$


Another good mathematical model for soap films is an $(\mathbf{M}, \varepsilon, \delta)$-minimal set, as was introduced by F. Almgren [1].

Definition $2.3([1])$. Let $\varepsilon$ be a function $\varepsilon(r)=C r^{\alpha}$ for some $0 \leq C<\infty$, $0<\alpha<1 / 3$ and $\delta>0$. $\Sigma \subset M$ is said to be an $(\mathbf{M}, \varepsilon, \delta)$-minimal set with respect to $\Gamma \subset M$ if $\Sigma$ is an $m$-dimensional rectifiable set and if, for every Lipschitz mapping $\phi: M \rightarrow M$ with the diameter $r=\operatorname{diam}(W \cup \phi(W))<\delta$,

$$
\mathcal{H}^{m}(\Sigma \cap W) \leq(1+\varepsilon(r)) \mathcal{H}^{m}(\phi(\Sigma \cap W)),
$$

where $W=\{x: \phi(x) \neq x\}$.

The following theorems for soap film-like surfaces in $\mathbb{R}^{3}$ are due to Jean Taylor.

Theorem 2.4 ([12]). The tangent cone of an (M,0, $\delta)$-minimal set $\Sigma$ at $p \in$ $\Sigma \backslash \Gamma$ is area minimizing with respect to the intersection with the unit sphere at $p$; Moreover, the plane, the $Y$-cone and the $T$-cone are the only possibilities for minimizing cones.

Theorem $2.5([12])$. An $(\mathbf{M}, \varepsilon, \delta)$-minimal set with respect to $\Gamma$ consists of $C^{1, \alpha}$ surfaces meeting smoothly in threes at $120^{\circ}$ angles along smooth curves, with these curves meeting in fours at angles of $\arccos (-1 / 3)$, away from $\Gamma$.

It was later proved by Kinderlehrer, Nirenberg, and Spruck that the surfaces which comprise $\Sigma$ are as smooth as the ambient manifold [9].

\section{Regularity of soap film-like surfaces in negatively curved spaces}

Let $M$ be an $n$-dimensional complete simply connected Riemannian manifold with sectional curvature bounded above by a nonpositive constant $-\kappa^{2}$. In this section we shall derive regularity theorems for soap film-like surfaces in $M$. The key step in the extension of the theorems to the variable curvature ambient space is to carry the data of $p \nVdash \Gamma$ over to the simply connected space form $\bar{M}$ of constant sectional curvature $-\kappa^{2}$, where $\kappa \geq 0$. To do this we construct a constant curvature metric on $p \circledast \Gamma$. For the sake of clarity, we give the definition as follows.

Definition 3.1 ([3], [5]). Let $\Gamma$ be an immersed piecewise $C^{1}$ curve in $M$. Let $\widehat{g}$ be a new metric on $p \times \Gamma$ with constant Gauss curvature $-\kappa^{2}$, such that the distance from $p$ remains the same as in the original metric $g$, and so does the arclength element of $\Gamma$.

We shall construct the new metric $\widehat{g}$ explicitly, and then extend Definition 3.1 to the case, where $\Gamma$ is a graph. Assuming for the moment that $\Gamma$ is a curve and that $\widehat{g}$ exists, one can see from the above definition that every geodesic from $p$ under $g$ remains a geodesic of equal length under $\widehat{g}$, the length of any arc of $\Gamma$ remains the same, and the angles between the tangent vector to $\Gamma$ and the geodesic from $p$ remain unchanged. Given a cone $C:=p \circledast \Gamma$, we shall denote 
by $\widehat{C}$ the two-dimensional Riemannian manifold $(C, \widehat{g})$, which will be singular at $p$.

Let $r(s)$ be the distance in $C$ from $p$ to the corresponding point of $\Gamma$ for an arc-length parameter $s$ of $\Gamma$. Then choose a point $\widehat{p} \in \mathbb{H}^{2}\left(-\kappa^{2}\right)$, and let a curve $\widehat{\Gamma}$ locally isometric to $\Gamma$ be traced out in $\mathbb{H}^{2}\left(-\kappa^{2}\right)$ so that the distance from $\widehat{p}$ equals $r(s)$. $\widehat{\Gamma}$ will close up, to form $\widehat{\Gamma}$ as a simply closed curve, in some Riemannian covering space $\widehat{M}^{2}$ of $\mathbb{H}^{2}\left(-\kappa^{2}\right) \backslash \widehat{p}$. Then $\widehat{C}$ can be written as $\widehat{p} \times \widehat{\Gamma}$ in $\widehat{M}$, along with the metric of $\widehat{M}$, which is singular at $\widehat{p}$ (see [3] for more details).

To prove the next proposition, we need the following well-known fact due to Euler (see [10]).

Lemma 3.2 (Euler). For a connected graph $\Gamma$ with even valence at each vertex, there is a continuous mapping of the circle to $\Gamma$ which traverses each edge exactly once.

Let $\Gamma^{\prime}$ be the double covering of the graph $\Gamma=\cup_{i=1}^{m} c_{i}$. By Lemma 3.2, we may choose an ordering for each edge such that $\Gamma^{\prime}$ is a piecewise smooth immersion of $\mathbb{S}^{1}$. In other words, $\Gamma^{\prime}=\cup_{j=1}^{2 m} c_{j}^{\prime}$, where each $c_{i}$ arises twice as one of the $c_{j}^{\prime}$ for $i=1, \ldots, m$. Then Definition 3.1 may be applied to $\Gamma^{\prime}$, to construct a metric $\widehat{g}$ of constant Gauss curvature on the disk $C^{\prime}=p * \Gamma^{\prime}$. Then each smooth surface $A_{i}=p \circledast c_{i}$ of the cone $C=p \circledast \Gamma$ is covered by two smooth "fans" $p * c_{j}^{\prime}$ and $p * c_{k}^{\prime}$ of $C^{\prime}(1 \leq j, k \leq 2 m)$. But $c_{j}^{\prime}$ and $c_{k}^{\prime}$ are copies of the $\operatorname{arc} c_{i}$ of $\Gamma$, so $p * c_{j}^{\prime}$ is isometric to $p * c_{k}^{\prime}$ using the metric $\widehat{g}$ of constant Gauss curvature for both. Thus, $p \nVdash c_{i}$ inherits the metric $\widehat{g}$ from either $p \nVdash c_{j}^{\prime}$ or $p \circledast c_{k}^{\prime}$, to form the singular cone $\widehat{C}$ with constant Gauss curvature on the interior of $p x_{i}, 1 \leq i \leq m$, and with a singular curve $\overline{p q}$ for each vertex $q$ of $\Gamma$. This completes the extension of Definition 3.1 for any graph $\Gamma$.

Proposition 3.3. Let $M$ be an n-dimensional simply connected Riemannian manifold with sectional curvature $K_{M} \leq-\kappa^{2} \leq 0$, and let $\Sigma \in \mathcal{S}_{\Gamma}$ be a strongly stationary surface in $M$. Then we have, for $p \in \Sigma \backslash \Gamma$,

$$
\Theta(\Sigma, p)<\Theta(\widehat{C}, p)
$$

unless $\Sigma$ is a cone over $p$ with totally geodesic faces of constant curvature $-\kappa^{2}$.

Proof. First let us assume $\widehat{K} \equiv-\kappa^{2}<0$; the case $\kappa=0$ will be treated similarly. Denote $r(x):=\operatorname{dist}(p, x)$, the distance function in $M$ from $p \in M$. Let $G(r(x)):=\log \tanh (\kappa r(x) / 2)$ be the Green's function for the two-dimensional hyperbolic plane $\mathbb{H}^{2}\left(-\kappa^{2}\right)$ with Gauss curvature $-\kappa^{2}$. On an immersed minimal surface $\Sigma_{i}$ in $M$, it follows from [5] that

$$
\triangle_{\Sigma} G(r)=2 \kappa^{2} \frac{\cosh \kappa r}{\sinh ^{2} \kappa r}\left(1-\left|\nabla_{\Sigma_{i}} r\right|^{2}\right) \geq 0 .
$$


Recall that $\Sigma=\cup_{i \in I} \Sigma_{i}$ and each $\Sigma_{i}$ is an immersed minimal surface. Integrating (3.1) over $\Sigma_{i} \backslash B_{\varepsilon}(p)$ gives

$$
0 \leq \int_{\Sigma_{i} \backslash B_{\varepsilon}(p)} \triangle_{\Sigma_{i}} G d A=\int_{\partial\left(\Sigma_{i} \backslash B_{\varepsilon}(p)\right)} \frac{\kappa}{\sinh \kappa r} \frac{\partial r}{\partial \nu_{\Sigma_{i}}} d s .
$$

Write $\Gamma=\cup_{j=1}^{m} c_{j}$. For a sufficiently small $\varepsilon>0$, let $C_{\varepsilon}:=C \backslash B_{\varepsilon}(p)=$ $(p \circledast \Gamma) \backslash B_{\varepsilon}(p)$ and $\Sigma_{i, \varepsilon}:=\Sigma_{i} \backslash B_{\varepsilon}(p)$. The divergence theorem yields

$$
0 \leq \int_{\Sigma_{i, \varepsilon}} \triangle_{\Sigma_{i, \varepsilon}} G d A=\int_{\partial \Sigma_{i, \varepsilon}} \frac{\kappa}{\sinh \kappa r} \frac{\partial r}{\partial \nu_{\Sigma_{i, \varepsilon}}} d s .
$$

Each boundary $\partial \Sigma_{i, \varepsilon}$ consists of three parts

$$
\partial \Sigma_{i, \varepsilon}=\left(\partial \Sigma_{i} \cap \Gamma\right) \cup\left(\partial B_{\varepsilon}(p) \cap \Sigma_{i}\right) \cup\left(\partial \Sigma_{i} \backslash\left(\Gamma \cup \overline{B_{\varepsilon}(p)}\right)\right) .
$$

Summing over $i \in I$, we get

$$
\begin{aligned}
0 \leq & \int_{\Gamma} \frac{\kappa}{\sinh \kappa r} \frac{\partial r}{\partial \nu_{\Sigma}} d s+\int_{\Sigma \cap \partial B_{\varepsilon}(p)} \frac{\kappa}{\sinh \kappa \varepsilon} \frac{\partial r}{\partial \nu_{\Sigma}} d s \\
& +\sum_{i \in I} \int_{\partial \Sigma_{i} \backslash\left(\Gamma \cup \overline{B_{\varepsilon}(p)}\right)} \frac{\kappa}{\sinh \kappa r} \frac{\partial r}{\partial \nu_{\Sigma_{i}}} d s,
\end{aligned}
$$

where $\nu_{\Sigma}$ is the outward unit conormal along the $\partial \Sigma_{\varepsilon}$. Since $\Sigma \in \mathcal{S}_{\Gamma}$, the last term vanishes by the balancing condition (2.1). Along $\Sigma \cap \partial B_{\varepsilon}(p), \frac{\partial r}{\partial \nu_{\Sigma}} \rightarrow-1$ uniformly as $\varepsilon \rightarrow 0$ and hence the second term converges to

$$
\lim _{\varepsilon \rightarrow 0}-\kappa \frac{\operatorname{Length}\left(\Sigma \cap \partial B_{\varepsilon}(p)\right)}{\sinh \kappa \varepsilon}=-2 \pi \Theta(\Sigma, p) .
$$

Therefore we obtain

$$
2 \pi \Theta(\Sigma, p) \leq \int_{\Gamma} \frac{\kappa}{\sinh \kappa r} \frac{\partial r}{\partial \nu_{\Sigma}} d s
$$

We repeat the same argument for $\widehat{C}=\widehat{p} \times \widehat{\Gamma}$ with $\widehat{C}$ instead of $\Sigma$ and $A_{j}=$ $\widehat{p} \circledast \widehat{c_{j}}$ instead of $\Sigma_{i}$. Thus the cone $\widehat{C}$ is the union of $A_{j}, 1 \leq j \leq m$. Since the Green's function $G(r)$ on $A_{j}$ satisfies [5, Lemma 3]

$$
\triangle_{A_{j}} G(r)=2 \kappa^{2} \frac{\cosh \kappa r}{\sinh ^{2} \kappa r}\left(1-\left|\nabla_{N} r\right|^{2}\right)
$$

it follows that $\triangle_{\widehat{C}} G(r)=0$. Applying the divergence theorem, we get

$$
0=\int_{A_{j} \backslash B_{\varepsilon}(p)} \triangle_{\widehat{C}} G d A=\int_{\partial\left(A_{j} \backslash B_{\varepsilon}(p)\right)} \frac{\kappa}{\sinh \kappa r} \frac{\partial r}{\partial \nu_{\widehat{C}}} d s .
$$

Each boundary $\partial\left(A_{j} \backslash B_{\varepsilon}(p)\right)$ consists of three parts:

$$
\partial\left(A_{j} \backslash B_{\varepsilon}(p)\right)=\left(\partial A_{j} \cap \Gamma\right) \cup\left(\partial B_{\varepsilon}(p) \cap A_{j}\right) \cup\left(\partial A_{j} \backslash\left(\Gamma \cup \overline{B_{\varepsilon}(p)}\right)\right) .
$$


Summing the above equation over $j=1, \ldots, m$, we obtain

$$
\begin{aligned}
0=\int_{\widehat{\Gamma}} \frac{\kappa}{\sinh \kappa r} \frac{\partial r}{\partial \nu_{C}} d s & +\int_{\widehat{C} \cap \partial B_{\varepsilon}(p)} \frac{\kappa}{\sinh \kappa \varepsilon} \frac{\partial r}{\partial \nu_{\widehat{C}}} d s \\
& +\sum_{j} \int_{\widehat{p} \mathbb{\Psi}\left(\partial \widehat{c_{j}}\right) \backslash B_{\varepsilon}(p)} \frac{\kappa}{\sinh \kappa r} \frac{\partial r}{\partial \nu_{A_{j}}} d s,
\end{aligned}
$$

where $\nu_{\widehat{C}}=\nu_{A_{j}}$ along $\partial A_{j}$ and $\nu_{A_{j}}$ is the outward unit conormal vector along the boundary $\partial A_{j}$. Since the conormal vector $\nu_{A_{j}}$ and $\bar{\nabla} r$ are perpendicular along $\widehat{p} \nVdash\left(\partial \widehat{c}_{j}\right)$, the last term vanishes. As $\varepsilon \rightarrow 0$, the second term converges to

$$
\lim _{\varepsilon \rightarrow 0}-\kappa \frac{\operatorname{Length}\left(\widehat{C} \cap B_{\varepsilon}(p)\right)}{\sinh \kappa \varepsilon}=-2 \pi \Theta(\widehat{C}, p) .
$$

Hence we have

$$
2 \pi \Theta(\widehat{C}, p)=\int_{\widehat{\Gamma}} \frac{\kappa}{\sinh \kappa r} \frac{\partial r}{\partial \nu_{\widehat{C}}} d s .
$$

On the other hand, from the definition of $\widehat{C}$ it is easy to see that

$$
\frac{\partial r}{\partial \nu_{C}}=\frac{\partial r}{\partial \nu_{\widehat{C}}}
$$

Since $\frac{\partial r}{\partial \nu_{C}} \geq \frac{\partial r}{\partial \nu_{\Sigma}}$ almost everywhere along $\Gamma$, the inequality (3.2) and the equations (3.3), (3.4) imply that

$$
2 \pi \Theta(\Sigma, p) \leq \int_{\Gamma} \frac{\kappa}{\sinh \kappa r} \frac{\partial r}{\partial \nu_{\Sigma}} d s \leq \int_{\widehat{\Gamma}} \frac{\kappa}{\sinh \kappa r} \frac{\partial r}{\partial \nu_{\widehat{C}}} d s=2 \pi \Theta(\widehat{C}, p) .
$$

If equality holds, then $\triangle_{\Sigma} G(r) \equiv 0$, which requires $\left|\nabla_{\Sigma} r\right| \equiv 1$. This can happen only when $\Sigma$ is totally geodesic. Similarly, using $G(r)=\log r$, we can prove the case where $\kappa=0$.

The following proposition was stated, but not proved, in Remark 3 of [5]. The version we will need in this section already appears as Proposition 4 of that paper; in the following section, we shall require this more general version.

Proposition 3.4. Let $g$ and $\widehat{g}$ be two continuous, piecewise $C^{2}$ metrics on the two-dimensional disk $C$, possibly singular at a certain point $p \in C$, such that the geodesics through $p$ (the "radial" geodesics) with respect to $g$ and $\widehat{g}$ are the same, with the same arc-length parameter along each radial geodesic. Assume that the arc length along the Lipschitz continuous boundary $\Gamma=\partial C$ is the same with respect to both metrics. For both metrics, assume there are no conjugate points along any radial geodesic. If the Gauss curvatures satisfy $\widehat{K} \geq K$ at each point of $C$, then the inward geodesic curvatures satisfy $\widehat{k} \leq k$ at each point of $\Gamma$.

Proof. As in Proposition 4 of [5], we consider normal Jacobi fields $V$ and $\widehat{V}$ along a unit-speed geodesic $\gamma(t)$ from $p=\gamma(0)$ to $q \in \Gamma$, such that $V(p)=$ $\widehat{V}(p)=0$ and $V(q)=\widehat{V}(q)$. This construction is possible since there are 
no conjugate points along $\gamma$ for either metric. Write $f(t)=|V(\gamma(t))|_{g}$ and $\widehat{f}(t)=|\widehat{V}(\gamma(t))|_{\widehat{g}}$ : both are positive except at $t=0$. At $q \in \Gamma$, the metrics are the same, so we have $|V(q)|_{g}=|\widehat{V}(q)|_{\widehat{g}}=1$. Then the inward curvatures $k_{0}$ and $\widehat{k}_{0}$ of the geodesic circle centered at $p$ satisfy

$$
k_{0}(\gamma(t))=\frac{f^{\prime}(t)}{f(t)} \quad \text { and } \quad \widehat{k}_{0}(\gamma(t))=\frac{\widehat{f}^{\prime}(t)}{\widehat{f}(t)} .
$$

The Jacobi equation $f^{\prime \prime}(t)+K(\gamma(t)) f(t)=0$ implies the Riccati equation $k_{0}^{\prime}+k_{0}^{2}=-K$, and similarly $\widehat{k}_{0}^{\prime}+\widehat{k}_{0}^{2}=-\widehat{K}$. Since $K \leq \widehat{K}$, these imply

$$
\left(k_{0}-\widehat{k}_{0}\right)^{\prime}+\left(k_{0}+\widehat{k}_{0}\right)\left(k_{0}-\widehat{k}_{0}\right) \geq 0,
$$

with $k_{0}-\widehat{k}_{0} \rightarrow 0$ at the point $\gamma(t)$ as $t \rightarrow 0$. Therefore $k_{0} \geq \widehat{k}_{0}$ everywhere along $\gamma$. Observe that the angles formed by $\Gamma$ and $\gamma$ with respect to $g$ and $\widehat{g}$ are the same. It now follows that the inward geodesic curvatures $k$ and $\widehat{k}$ of $\Gamma$ with respect to $g$ and $\widehat{g}$ satisfy $\widehat{k} \leq k$ (see the proof of Proposition 4 of [5]).

Proposition 3.5 ([5, Proposition 5]). Let $\Gamma$ be a $C^{2}$ curve in $M$ and let $C$ be the cone $p * \Gamma$. If $\widehat{C}$ is the cone $C$ equipped with the constant curvature metric $\widehat{g}$, as in Definition 3.1 above, then $\Theta(C, p) \leq \Theta(\widehat{C}, p)$ and $\operatorname{Area}(C) \leq \operatorname{Area}(\widehat{C})$.

Now we prove the following Gauss-Bonnet formula for two-dimensional cones in a nonpositively curved manifold.

Proposition 3.6 (Gauss-Bonnet formula). Let $p$ be a point in $M \backslash \Gamma$ for a graph $\Gamma$ with edges $c_{1}, \ldots, c_{m}$. Let $\widehat{C}=(C, \widehat{g})$ for $C=p * \Gamma$. Then we have

$$
2 \pi \Theta(\widehat{C}, p)+\kappa^{2} \operatorname{Area}(\widehat{C})=-\sum_{i=1}^{m} \int_{\widehat{c_{i}}} \widehat{k} d s+\sum_{i=1}^{m} \sum_{j=0,1}\left(\frac{\pi}{2}-\measuredangle_{q_{j}^{i}}\left(T_{i}\left(q_{j}^{i}\right), \overline{q_{j}^{i} p}\right)\right),
$$

where $\widehat{k}$ is the geodesic curvature of $\Gamma$ in $\widehat{C}$ and $\angle_{q_{j}^{i}}\left(T_{i}\left(q_{j}^{i}\right), \overline{q_{j}^{i} p}\right)$ is the angle at $q_{j}^{i}$ between the tangent vector $T_{i}\left(q_{j}^{i}\right)$ to $c_{i}$ and the geodesic $\overline{q_{j}^{i} p}$.

Proof. Suppose first that $\Gamma$ is a smooth closed curve in $M^{n}$ but not necessarily simple, and $p$ is a point of $M^{n} \backslash \Gamma$. Choose a sufficiently small $\varepsilon>0$ such that $\overline{B_{\varepsilon}(p)}$ does not intersect $\Gamma$. Let $A=\widehat{C} \backslash B_{\varepsilon}(p)$ be the annular region between $\Gamma$ and $\partial B_{\varepsilon}(p) \cap \widehat{C}$. The Gauss-Bonnet formula says that

$$
\int_{A} \widehat{K} d A-\int_{\Gamma} \vec{k} \cdot \nu_{C} d s-\int_{\partial B_{\varepsilon}(p) \cap\left(p \Psi_{\Gamma}\right)} \vec{k} \cdot \nu_{\widehat{C}} d s=2 \pi \chi(A),
$$

where $\chi(A)$ is the Euler characteristic of $A$ and $\widehat{K}=-\kappa^{2}$ is the intrinsic Gauss curvature of $\widehat{C}$. Since $A$ is an immersed annulus, we have $\chi(A)=0$. Along $\partial B_{\varepsilon}(p) \cap \widehat{C}$, we have $\nu_{\widehat{C}}=-\bar{\nabla} r$ and $\vec{k} \cdot \nu_{\widehat{C}} \equiv \widehat{k}_{0}(\varepsilon)$, where $k_{0}(\varepsilon)=\kappa \cot \kappa \varepsilon$ 
is its geodesic curvature, as in Proposition 3.4, and $r$ is the distance from $\widehat{p}$ in $M$. Note that

$$
\begin{aligned}
\lim _{\varepsilon \rightarrow 0} \int_{\partial B_{\varepsilon}(p) \cap \widehat{C}} \vec{k} \cdot \nu_{\widehat{C}} & =\lim _{\varepsilon \rightarrow 0} \widehat{k}_{0}(\varepsilon) \operatorname{Length}\left(\partial B_{\varepsilon}(p) \cap \widehat{C}\right) \\
& =2 \pi \Theta(\widehat{C}, p) .
\end{aligned}
$$

Letting $\varepsilon \rightarrow 0$, we get

$$
-\kappa^{2} \operatorname{Area}(\widehat{C})-\int_{\Gamma} \vec{k} \cdot \nu_{\widehat{C}}-2 \pi \Theta(\widehat{C}, p)=0 .
$$

Finally, in the general case of a graph $\Gamma \subset M^{n}$, we apply Lemma 3.2 to show that the double cover of $\Gamma$ can be considered as a piecewise smooth immersion $\Gamma^{\prime}$ of $\mathbb{S}^{1}$.

Since $\Gamma^{\prime}$ is a piecewise-smooth immersion of the circle, we may apply equation (3.5) to $\Gamma^{\prime}$, and conclude that

$$
\kappa^{2} \operatorname{Area}\left(\widehat{C}^{\prime}\right)+\int_{\Gamma^{\prime}} \vec{k} \cdot \nu_{C} d s+2 \pi \Theta\left(\widehat{C}^{\prime}, p\right)=0 .
$$

Let $q_{0}^{i}, q_{1}^{i}$ be the end points of the smooth segment $c_{i}$ for $i=1, \ldots, m$. We denote $q_{j}^{i} \sim q_{\ell}^{k}$ if they represent the same point of $M$ where $c_{i}$ and $c_{k}$ meet. Denote $A_{j}^{\prime}=\widehat{p} \nVdash c_{j}^{\prime}$ with the metric of constant curvature. Then we see that $\widehat{C}^{\prime}=\cup_{j=1}^{2 m} A_{j}^{\prime}=\widehat{p} \times \Gamma^{\prime}$.

Similar arguments as in the above smooth case show that

$2 \pi \Theta\left(\widehat{C}^{\prime}, p\right)=-\kappa^{2} \operatorname{Area}(A)-\sum_{i=1}^{m} \int_{c_{i}} \vec{k} \cdot \nu_{c_{i}} d s+\sum_{i=1}^{m} \sum_{\ell=0,1}\left(\frac{\pi}{2}-\angle_{q_{\ell}^{i}}\left(T_{i}\left(q_{\ell}^{i}\right), \overline{q_{\ell}^{i} p}\right)\right)$.

Indeed, the last term is equal to the sum over vertices of the sum of the exterior angles of the piecewise smooth curve $\Gamma^{\prime}$ at the vertex $q_{1}^{i} \sim q_{0}^{k}$. To see this, suppose that $c_{i}$ and $c_{k}$ are the consecutive edges in $\Gamma$ joined at $q_{1}^{i} \sim q_{0}^{k}$; then

$$
\begin{aligned}
& \left(\frac{\pi}{2}-\angle_{q_{1}^{i}}\left(T_{i}\left(q_{1}^{i}\right), \overline{q_{1}^{i} p}\right)\right)+\left(\frac{\pi}{2}-\angle_{q_{0}^{k}}\left(T_{k}\left(q_{0}^{k}\right), \overline{q_{0}^{k} p}\right)\right) \\
= & \pi-\left(\angle_{q_{1}^{i}}\left(T_{i}\left(q_{1}^{i}\right), \overline{q_{1}^{i} p}\right)+\angle_{q_{0}^{k}}\left(T_{k}\left(q_{0}^{k}\right), \overline{q_{0}^{k} p}\right)\right) \\
= & \pi-\angle_{q_{1}^{i}}\left(T_{i}\left(q_{1}^{i}\right), T_{k}\left(q_{0}^{k}\right)\right),
\end{aligned}
$$

which is the exterior angle between $c_{i}$ and $c_{k}$ at $q_{1}^{i} \sim q_{0}^{k}$, relative to the constantcurvature cone $\widehat{C}^{\prime}$.

Therefore

$$
\begin{aligned}
2 \pi \Theta\left(\widehat{C}^{\prime}, p\right)= & -\kappa^{2} \operatorname{Area}\left(\widehat{C}^{\prime}\right)-\sum_{i=1}^{2 m} \int_{c_{i}^{\prime}} \vec{k} \cdot \nu_{\widehat{c}_{i}^{\prime}} d s \\
& +\sum_{i=1}^{2 m} \sum_{j=0,1}\left(\frac{\pi}{2}-\angle_{q_{j}^{i}}\left(T_{i}\left(q_{j}^{i}\right), \overline{q_{j}^{i} p}\right)\right) .
\end{aligned}
$$


For the edges $c_{i_{1}}$ and $c_{i_{2}}$ which represent the same edge $c_{i}$ of $\Gamma$, we have

$$
\int_{\widehat{c}_{i}} \vec{k} \cdot \nu_{\widehat{C}} d s=\int_{\widehat{c}_{i_{1}}} \vec{k} \cdot \nu_{\widehat{C}} d s=\int_{\widehat{c}_{i_{2}}} \vec{k} \cdot \nu_{\widehat{C}} d s .
$$

We also note that the exterior angle appears twice and

$$
\operatorname{Area}\left(\widehat{p} \circledast \widehat{\Gamma}^{\prime}\right)=2 \operatorname{Area}(\widehat{p} * \widehat{\Gamma})
$$

Therefore by dividing both sides of equation (3.7) by two, we obtain

$$
\begin{aligned}
2 \pi \Theta(\widehat{C}, p)+\kappa^{2} \operatorname{Area}(\widehat{C})= & -\sum_{i=1}^{m} \int_{\widehat{c}_{i}} \vec{k} \cdot \nu_{\widehat{c}_{i}} d s \\
& +\sum_{i=1}^{m} \sum_{j=0,1}\left(\frac{\pi}{2}-\measuredangle_{q_{j}^{i}}\left(T_{i}\left(q_{j}^{i}\right), \overline{q_{j}^{i} p}\right)\right)
\end{aligned}
$$

which completes the proof.

Definition 3.7. Define the minimum cone area $\mathcal{A}(\Gamma)$ of a graph $\Gamma \subset M$ as

$$
\mathcal{A}(\Gamma):=\inf _{p \in \operatorname{Conv}(\Gamma)} \operatorname{Area}(p \circledast \Gamma) .
$$

Theorem 3.8. Let $M$ be an $n$-dimensional complete simply connected Riemannian manifold whose sectional curvature is bounded above by a nonpositive constant $-\kappa^{2}$. Let $\Gamma$ be a graph in $M$ with $T C(\Gamma) \leq 3 \pi+\kappa^{2} \mathcal{A}(\Gamma)$ and let $\Sigma \in \mathcal{S}_{\Gamma}$ be a strongly stationary surface with respect to $\Gamma$ in $M$. Then $\Sigma$ is either an embedded surface or a subset of the $Y$-singularity cone with totally geodesic faces having constant Gauss curvature $-\kappa^{2}$.

Proof. For a point $p$ on $\Sigma \backslash \Gamma$, Proposition 3.3 says that

$$
2 \pi \Theta(\Sigma, p) \leq 2 \pi \Theta(\widehat{C}, p) .
$$

Applying Proposition 3.4, Proposition 3.5, and Proposition 3.6, we have

$$
\begin{aligned}
2 \pi \Theta(\widehat{C}, p) & =-\kappa^{2} \operatorname{Area}(\widehat{C})-\int_{\Gamma} \widehat{k} d s+\sum_{i=1}^{m} \sum_{j=0,1}\left(\frac{\pi}{2}-\angle_{q_{j}^{i}}\left(T_{i}\left(q_{j}^{i}\right), \overline{q_{j}^{i} p}\right)\right) \\
& \leq-\kappa^{2} \operatorname{Area}(p \circledast \Gamma)-\int_{\Gamma} k d s+\sum_{i=1}^{m} \sum_{j=0,1}\left(\frac{\pi}{2}-\angle_{q_{j}^{i}}\left(T_{i}\left(q_{j}^{i}\right), \overline{q_{j}^{i} p}\right)\right) \\
& \leq T C(\Gamma)-\kappa^{2} \operatorname{Area}(p \circledast \Gamma) .
\end{aligned}
$$

Therefore the assumption on the cone total curvature of $\Gamma$ implies that

$$
2 \pi \Theta(\Sigma, p) \leq 3 \pi
$$

If $\Theta(\Sigma, p)<\frac{3}{2}$ for any $p \in \Sigma \backslash \Gamma$, then $\Sigma$ is an embedded surface. Otherwise, we have $\Theta(\Sigma, p)=\frac{3}{2}$ which means that $\Sigma$ is a cone with vertex $p$ and totally geodesic faces. Since the only stationary cone with density $\frac{3}{2}$ is the $Y$-cone, $\Sigma$ is a subset of the $Y$-cone. 
Theorem 3.9. Let $M^{3}$ be a 3-dimensional complete simply connected Riemannian manifold whose sectional curvature is bounded above by a nonpositive constant $-\kappa^{2}$. Let $\Sigma \in \mathcal{S}_{\Gamma}$ be embedded as an $(\mathbf{M}, 0, \delta)$-minimizing set with respect to a graph $\Gamma$ in $M^{3}$. If $T C(\Gamma) \leq 2 \pi C_{T}+\kappa^{2} \mathcal{A}(\Gamma)$, then $\Sigma$ can have only $Y$ singularities unless $\Sigma$ is a subset of the $T$ stationary cone with totally geodesic faces.

Proof. As in the proof of the above theorem, we can see that at $p \in \Sigma \backslash \Gamma$

$$
\begin{aligned}
2 \pi \Theta(\Sigma, p) & \leq 2 \pi \Theta(\widehat{C}, p) \\
& \leq T C(\Gamma)-\kappa^{2} \operatorname{Area}(p \circledast \Gamma) \leq 2 \pi C_{T} .
\end{aligned}
$$

By Theorem 2.4, we see that for $p \in \Sigma \backslash \Gamma$, if $\Theta(\Sigma, p)=1$, then $\Sigma$ is a plane. If $\Theta(\Sigma, p)=\frac{3}{2}$, then $\Sigma$ is a subset of the $Y$-cone. If $\Theta(\Sigma, p)=C_{T}$, then $\Sigma$ is a subset of the $T$-cone.

\section{Regularity of soap film-like surfaces in spaces with bounded diameter}

Let $M^{n}$ be a simply-connected Riemannian manifold with diameter $\leq \frac{\pi}{b}$ and sectional curvatures $K_{M} \leq b^{2}$, where $b>0$. Recall that any two points of $M$ are connected by a unique geodesic in $M$. As in Section 3, we may define the convex hull $\operatorname{Conv}(S)$ of a subset $S$ of $M$. It is not difficult to see that $\operatorname{Conv}(S)$ is the intersection of closed geodesically convex sets containing $S$, and it follows that if $\Sigma \in \mathcal{S}_{\Gamma}$ is strongly stationary with respect to a graph $\Gamma$ in $M^{n}$, then $\Sigma \subset \operatorname{Conv}(\Gamma)$.

Definition 4.1. Let $\widehat{g}$ be a new metric on $C=p \circledast \Gamma$ with constant Gauss curvature $b^{2}$ such that the distance from $p$ remains the same as in the original metric $g$, and so does the arclength element of $\Gamma$.

As in Section 3, every geodesic from $p$ under $g$ remains a geodesic of equal length under $\hat{g}$, the length of any arc of $\Gamma$ remains the same, and the angles between the tangent vector to $\Gamma$ and the geodesic from $p$ remain unchanged. $\widehat{C}=(C, \widehat{g})$ may be constructed as in Section 3, with the two-dimensional sphere of radius $1 / b$ replacing $\mathbb{H}^{2}\left(-\kappa^{2}\right)$.

Proposition 4.2. Let $\Sigma \in \mathcal{S}_{\Gamma}$ be a strongly stationary surface in $M^{n}$ and let $p$ be a point in $\Sigma \backslash \Gamma$. Then

$$
\Theta(\Sigma, p)<\Theta(\widehat{C}, p)
$$

unless $\Sigma$ is a cone over $p$ with totally geodesic faces and constant Gauss curvature $b^{2}$.

Proof. The proof involves computations analogous to Proposition 3.3 on $\Sigma$ and on $\widehat{C}$, related to the well-known monotonicity inequality. 
Let $G(r(x))=\log \tan (b r(x) / 2)$ be the Green's function of the two-dimensional sphere of constant curvature $b^{2}$, where $r(x)$ is the distance from $p$ in $M^{n}$. On an immersed minimal surface $\Sigma_{i}$ in $M$, it follows from [4] that

$$
\triangle_{\Sigma_{i}} G(r)=2 \frac{b^{2} \cos b r}{\sin ^{2} b r}\left(1-\left|\nabla_{\Sigma_{i}} r\right|^{2}\right) \geq 0
$$

Recall that $\Sigma=\cup_{i \in I} \Sigma_{i} \in \mathcal{S}_{\Gamma}$ and each $\Sigma_{i}$ is minimal. Integrating (4.1) over each $\Sigma_{i} \backslash B_{\varepsilon}(p)$ for sufficiently small $\varepsilon>0$ gives

$$
0 \leq \int_{\Sigma_{i} \backslash B_{\varepsilon}(p)} \triangle_{\Sigma_{i}} G d A=\int_{\partial\left(\Sigma_{i} \backslash B_{\varepsilon}(p)\right)} \frac{b}{\sin b r} \frac{\partial r}{\partial \nu_{\Sigma_{i}}} d s .
$$

Since $\partial\left(\Sigma_{i} \backslash B_{\varepsilon}(p)\right)=\left(\partial \Sigma_{i} \cap \Gamma\right) \cup\left(\partial B_{\varepsilon}(p) \cap \Sigma_{i}\right) \cup\left(\partial \Sigma_{i} \backslash\left(\Gamma \cup \overline{B_{\varepsilon}(p)}\right)\right)$, summing over $i$ gives

$$
\begin{aligned}
0 \leq & \int_{\Gamma} \frac{b}{\sin b r} \frac{\partial r}{\partial \nu_{\Sigma}} d s+\int_{\Sigma \cap \partial B_{\varepsilon}(p)} \frac{b}{\sin b \varepsilon} \frac{\partial r}{\partial \nu_{\Sigma}} d s \\
& +\sum_{i \in I} \int_{\partial \Sigma_{i} \backslash\left(\Gamma \cup \overline{B_{\varepsilon}(p)}\right)} \frac{b}{\sin b r} \frac{\partial r}{\partial \nu_{\Sigma_{i}}} d s .
\end{aligned}
$$

Applying the balancing condition (2.1) at each point $p \in \partial \Sigma_{i} \backslash \Gamma$, one can see that the last term vanishes.

Since along $\Sigma \cap \partial B_{\varepsilon}(p), \frac{\partial r}{\partial \nu_{\Sigma}} \rightarrow-1$ uniformly as $\varepsilon \rightarrow 0$, the second term converges to

Hence we have

$$
\lim _{\varepsilon \rightarrow 0}-\frac{b \text { Length }\left(\Sigma \cap \partial B_{\varepsilon}(p)\right)}{\sin b \varepsilon}=-2 \pi \Theta(\Sigma, p) .
$$

$$
2 \pi \Theta(\Sigma, p) \leq \int_{\Gamma} \frac{b}{\sin b r} \frac{\partial r}{\partial \nu_{\Sigma}} d s
$$

Similarly, one can estimate the density $\Theta(\widehat{C}, p)$ at $p$ of the cone $p \circledast \Gamma$ with the metric $\widehat{g}$ of constant Gauss curvature $b^{2}$. Because $\Gamma=\cup_{j=1}^{m} c_{j}$, where each arc $c_{j}$ is $C^{2}$ and $C^{1}$ up to the end points, the cone $C=p \circledast \Gamma$ can be represented as

$$
C=p * \Gamma=\cup \overline{A_{j}}=\cup\left(A_{j} \cup \partial A_{j}\right),
$$

where $A_{j}=p \nVdash c_{j}$ is a $C^{2}$ surface, $C^{1}$ up to its boundary, possibly not immersed. Write $\widehat{C}=\cup \overline{A_{j}}$. On each $\widehat{A}_{j}$, it follows from [4] that the Green's function $G(r)$ is harmonic because

$$
\triangle_{\widehat{C}} G(r)=2 \frac{b^{2} \cos b r}{\sin ^{2} b r}\left(1-\left|\nabla_{\widehat{C}} r\right|^{2}\right)=0 .
$$

The divergence theorem yields

$$
0=\int_{A_{j} \backslash B_{\varepsilon}(p)} \triangle_{\widehat{C}} G d A=\int_{\partial\left(A_{j} \backslash B_{\varepsilon}(p)\right)} \frac{b}{\sin b r} \frac{\partial r}{\partial \nu_{\widehat{C}}} d s .
$$

As before, each boundary $\partial\left(A_{j} \backslash B_{\varepsilon}(p)\right)$ consists of three parts. 
Summing the above equation (4.4) over $j=1, \ldots, m$, since $\nu_{\widehat{C}}=\nu_{C}$ along $\Gamma$, we have

$$
\begin{aligned}
0= & \int_{\Gamma} \frac{b}{\sin b r} \frac{\partial r}{\partial \nu_{\widehat{C}}} d s+\int_{C \cap \partial B_{\varepsilon}(p)} \frac{b}{\sin b \varepsilon} \frac{\partial r}{\partial \nu_{\widehat{C}}} d s \\
& +\sum_{j} \int_{\left(p \aleph c_{j}\right) \backslash B_{\varepsilon}(p)} \frac{b}{\sin b r} \frac{\partial r}{\partial \nu_{\widehat{A}_{j}}} d s,
\end{aligned}
$$

where $\nu_{A_{j}}$ denotes the outward unit conormal vector along the boundary $\partial A_{j}$ and $\nu_{C}(q)$ is defined to be $\sum_{j=1}^{m}\left\{\nu_{A_{j}}(q): q \in \partial A_{j}\right\}$. Note that along $p \nVdash\left(\partial c_{j}\right)$, the conormal vector $\nu_{\widehat{A}_{j}}$ and $\bar{\nabla} r$ are perpendicular, i.e. $\frac{\partial r}{\partial \nu_{\widehat{A}_{j}}} \equiv 0$. Hence the last term vanishes in the above equation. Since $\frac{\partial r}{\partial \nu_{\widehat{C}}} \rightarrow 1$ as $\varepsilon \rightarrow 0$ along $C \cap \partial B_{\varepsilon}(p)$ and hence

$$
-\frac{b \text { Length }\left(C \cap B_{\varepsilon}(p)\right)}{\sin b \varepsilon} \rightarrow-2 \pi \Theta(C, p),
$$

one can see that the second term converges to $-2 \pi \Theta(C, p)$. Therefore we have

$$
2 \pi \Theta(C, p)=\int_{\Gamma} \frac{b}{\sin b r} \frac{\partial r}{\partial \nu_{C}} d s
$$

Since $\frac{\partial r}{\partial \nu_{C}} \geq \frac{\partial r}{\partial \nu_{\Sigma}}$ almost everywhere along $\Gamma$, it follows from (4.2) and (4.5) that

$$
2 \pi \Theta(\Sigma, p) \leq \int_{\Gamma} \frac{b}{\sin b r} \frac{\partial r}{\partial \nu_{\Sigma}} d s \leq \int_{\Gamma} \frac{b}{\sin b r} \frac{\partial r}{\partial \nu_{C}} d s=2 \pi \Theta(C, p) .
$$

If equality holds, then $\Delta_{\Sigma} G \equiv 0$. So we should have $\left|\nabla_{\Sigma} r\right| \equiv 1$ by (4.3), which can happen only when $\Sigma$ is totally geodesic; and $\triangle_{\Sigma} G \equiv 0$ [4].

We prove the following Gauss-Bonnet formula for singular two-dimensional cones in $M^{n}$.

Proposition 4.3 (Gauss-Bonnet formula). Let $p$ be a point of $M^{n} \backslash \Gamma$ for a graph $\Gamma=\cup c_{i}$. Let $\widehat{C}=(C, \widehat{g})$ for $C=p \times \Gamma$. Then we have

$$
2 \pi \Theta(\widehat{C}, p)=b^{2} \operatorname{Area}(\widehat{C})-\sum_{i=1}^{m} \int_{c_{i}} \vec{k} \cdot \nu_{c_{i}} d s+\sum_{i=1}^{m} \sum_{j=0,1}\left(\frac{\pi}{2}-\angle_{q_{j}^{i}}\left(T_{i}\left(q_{j}^{i}\right), \overline{q_{j}^{i} p}\right)\right),
$$

where $\vec{k}$ is the curvature vector of $c_{i}$ in $\widehat{C}, \nu_{c_{i}}$ is the outward unit conormal vector to $p \circledast \Gamma$ along $c_{i}, \angle_{q_{j}^{i}}\left(T_{i}\left(q_{j}^{i}\right), \overline{q_{j}^{i} p}\right)$ is the angle at $q_{j}^{i}$ between the tangent vector $T_{i}\left(q_{j}^{i}\right)$ to $c_{i}$ and the geodesic $\overline{q_{j}^{i} p}$.

Proof. Similar arguments as in the proof of Proposition 3.6 give the desired result.

As a consequence of the density comparison (Proposition 4.2) and the GaussBonnet Theorem for two-dimensional cones in $M^{n}$ (Proposition 4.3), there follows: 
Theorem 4.4. Let $M^{n}$ be a manifold with $K_{M} \leq b^{2}$ and diameter $\leq \frac{\pi}{b}$. Let $\Gamma$ be a graph in $M^{n}$ and let $\Sigma \in \mathcal{S}_{\Gamma}$ be a strongly stationary surface with respect to $\Gamma$ in $M^{n}$. Then for $p \in M^{n}$ we have

$$
2 \pi \Theta(\Sigma, p) \leq T C(\Gamma)+b^{2} \operatorname{Area}(p \circledast \Gamma) .
$$

Definition 4.5. Define the maximum cone area $\overline{\mathcal{A}}(\Gamma)$ of a graph $\Gamma \subset M^{n}$ as

$$
\overline{\mathcal{A}}(\Gamma):=\sup _{p \in \operatorname{Conv}(\Gamma)} \operatorname{Area}(p \circledast \Gamma) .
$$

Now we state and prove the regularity theorems for soap film-like surfaces spanning graphs with small cone total curvature in a manifold of bounded diameter.

Theorem 4.6. Let $M^{n}$ be a manifold with $K_{M} \leq b^{2}$ and diameter $\leq \frac{\pi}{b}$, and let $\Gamma$ be a graph with cone total curvature $T C(\Gamma) \leq 3 \pi-b^{2} \overline{\mathcal{A}}(\Gamma)$. Let $\Sigma \in \mathcal{S}_{\Gamma}$ be a strongly stationary surface with respect to $\Gamma$ in $M^{n}$. Then $\Sigma$ is either an embedded surface or a subset of the $Y$-singular cone formed by three totally geodesic surfaces of constant Gauss curvature $b^{2}$.

Proof. At $p \in \Sigma \backslash \Gamma$, we have

$$
2 \pi \Theta(\Sigma, p) \leq 2 \pi \Theta(p * \Gamma, p) \leq T C(\Gamma)+b^{2} \operatorname{Area}(p * \Gamma) \leq 3 \pi .
$$

If $\Theta(\Sigma, p)<\frac{3}{2}$, then $\Sigma$ is an embedded surface. Otherwise, $\Theta(\Sigma, p)=\frac{3}{2}$ which means that $\Sigma$ is a cone with vertex $p$ and totally geodesic faces. Since the only stationary cone with density $\frac{3}{2}$ is the $Y$-cone, $\Sigma$ is a subset of the $Y$-cone.

Given a strongly stationary surface $\Sigma$ in $\mathcal{S}_{\Gamma}$, we have seen that the first nontrivial upper bound for the density of $\Sigma$, other than 1 , is $3 / 2$. In order to find a larger upper bound for density, we consider the case of ambient dimension $n=3$. Note that the tangent cone of a strongly stationary soap film-like surface in $M^{3}$ is exactly the same as in Euclidean space $\mathbb{R}^{3}$. Since there are only three area minimizing cones in $\mathbb{R}^{3}[2]$, the only possible candidate for a larger bound for density is the $T$-singularity cone.

Theorem 4.7. Let $M^{n}$ be a manifold with $K_{M} \leq b^{2}$ and diameter $\leq \frac{\pi}{b}$. Let $\Gamma$ be a graph in $M^{3}$ with $T C(\Gamma) \leq 2 \pi C_{T}-b^{2} \overline{\mathcal{A}}(\Gamma)$ and let $\Sigma \in \mathcal{S}_{\Gamma}$ be embedded as an $(\mathbf{M}, 0, \delta)$-minimizing set with respect to $\Gamma$. Then $\Sigma$ can have only $Y$ singularities unless $\Sigma$ is a subset of the T stationary cone with totally geodesic faces.

Proof. For any point $p \in \Sigma \backslash \Gamma$ we have

$$
2 \pi \Theta(\Sigma, p) \leq 2 \pi \Theta(p \circledast \Gamma, p) \leq T C(\Gamma)+b^{2} \operatorname{Area}(p \circledast \Gamma) \leq 2 \pi C_{T} .
$$

It follows from Theorem 2.4 that the tangent cone of an $(\mathbf{M}, 0, \delta)$-minimal set $\Sigma$ at $p$ is area-minimizing with respect to the intersection with the unit sphere centered at $p$, and that the plane, the $Y$-cone and the $T$-cone are the only possibility for a area-minimizing tangent cone. Hence for $p \in \Sigma \backslash \Gamma$, if 
$\Theta(\Sigma, p)=1$, then $\Sigma$ is a plane. If $\Theta(\Sigma, p)=\frac{3}{2}$, then $\Sigma$ is the $Y$-cone. Moreover if $\Theta(\Sigma, p)=C_{T}$, then $\Sigma$ is the $T$-cone.

Remark. We do not know whether in general an $(\mathbf{M}, 0, \delta)$-minimizing set with respect to a graph is an element of the class $\mathcal{S}_{\Gamma}$ or not. Note that an $(\mathbf{M}, 0, \delta)$ minimal set $\Sigma$ in $\mathcal{S}_{\Gamma}$ with variational boundary $\Gamma$ is strongly stationary with respect to $\Gamma$. However the converse is not true in general. For instance, consider the cone over the 1-skeleton $\Gamma$ of the cube. It is strongly stationary with respect to $\Gamma$, but not an $(\mathbf{M}, 0, \delta)$-minimal set (see [12]).

For our final theorem, we return to the context of minimal branched immersions of surfaces, as treated in [6] and in [5]. Note that the density of such a surface $\Sigma$ in $M^{n}$ must be an integer $\geq 1$ at each point. At a branch point, the density is $\geq 2$; at a point $p \in M$ of self-intersection, the density $\Theta(\Sigma, p)$ equals the number of pieces of surface which intersect at $p$. In the same spirit as the theorems above, which refer to $C_{Y}=\frac{3}{2}$ and $C_{T} \approx 1.8245$, we may define $C_{X}=2$, the minimum density at a self-intersection point or branch point of a branched immersion.

Theorem 4.8. Let $M^{n}$ be a manifold with $K_{M} \leq b^{2}$ and diameter $\leq \frac{\pi}{b}$. Let $\Gamma$ be a simple closed curve in $M^{3}$ with $T C(\Gamma)<2 \pi C_{X}-b^{2} \overline{\mathcal{A}}(\Gamma)$, and let $\Sigma$ be a branched immersion of a compact surface into $M^{n}$ with boundary $\Gamma$. Then $\Sigma$ is embedded.

The proof is analogous to the proofs of Theorems 4.6 and 4.7, showing that the density of $\Sigma$ at any point of the convex hull of $\Gamma$ is less than two. This result is Theorem 1 of [5] in the specific case where $M$ is the $n$-dimensional hemisphere of constant sectional curvature $b^{2}$.

\section{References}

[1] F. Almgren, Existence and regularity almost everywhere of solutions to elliptic variational problems with constraints, Mem. Amer. Math. Soc. 4 (1976), no. 165, viii+199 pp.

[2] F. Almgren and J. Taylor, The geometry of soap films and soap bubbles, Scientific American 235 (1976), 82-93.

[3] J. Choe, The isoperimetric inequality for minimal surfaces in a Riemannian manifold, J. Reine Angew. Math. 506 (1999), 205-214.

[4] J. Choe and R. Gulliver, Isoperimetric inequalities on minimal submanifolds of space forms, Manuscripta Math. 77 (1992), no. 2-3, 169-189.

[5] _ Embedded minimal surfaces and total curvature of curves in a manifold, Math. Res. Lett. 10 (2003), no. 2-3, 343-362.

[6] T. Ekholm, B. White, and D. Wienholtz, Embeddedness of minimal surfaces with total

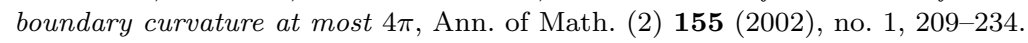

[7] H. Federer, Geometric Measure Theory, Springer-Verlag, New York, 1969.

[8] R. Gulliver and S. Yamada, Area density and regularity for soap film-like surfaces spanning graphs, Math. Z. 253 (2006), no. 2, 315-331.

[9] D. Kinderlehrer, L. Nirenberg, and J. Spruck, Regularity in elliptic free boundary problems, J. Analyse Math. 34 (1978), 86-119

[10] O. Ore, Graphs and Their Uses, Random House, New York, 1963. 
[11] L. Simon, Lectures on geometric measure theory, Proceedings of the Centre for Mathematical Analysis, Australian National University, 3. Australian National University, Centre for Mathematical Analysis, Canberra, 1983. vii+272 pp.

[12] J. Taylor, The structure of singularities in soap-bubble-like and soap-film-like minimal surfaces, Ann. of Math. (2) 103 (1976), no. 3, 489-539.

Robert Gulliver

SCHOOL OF Mathematics

UNIVERSITY OF MINNESOTA

MiNNEAPOLIS, MN 55455, USA

E-mail address: gulliver@math.umn.edu

SUNG-HO PARK

Graduate School of Education

HANKUK University of Foreign Studies

270 Imun-dong, Dongdaemun-Gu

SEOUl 130-791, KoreA

E-mail address: sunghopark@hufs.ac.kr

JUNCHEOL PYO

Department of Mathematics

Seoul National University

SEOUl 151-742, KoreA

E-mail address: jcpyo@snu.ac.kr

KeOMKYo SEO

Department of Mathematics

SOOKMYUng WoMEN'S UNIVERSITY

SeOul 140-742, KoreA

E-mail address: kseo@sookmyung.ac.kr 